\section{JTI}

JOURNAL OF

TRAUMA AND INJURY

\title{
Role of the Neutrophil-to-Lymphocyte Ratio at the Time of Arrival at the Emergency Room as a Predictor of Rhabdomyolysis in Severe Trauma Patients
}

Received: March 31, 2020

Revised: May 25, 2020

Accepted: June 2, 2020

\section{Correspondence to}

Kyung Hoon Sun, M.D., Ph.D.

Department of Emergency Medicine Chosun University Hospital,

365 Philmun-daero, Dong-gu, Gwangju 61453, Korea

Tel: +82-62-220-3285

Fax: +82-62-224-3501

E-mail:skhkorea@hanmail.net

\author{
Jin Chul Bae, M.D., Kyung Hoon Sun, M.D., Ph.D., Yong Jin Park, M.D., Ph.D. \\ Department of Emergency Medicine, College of Medicine, Chosun University, Gwangju, \\ Korea
}

Purpose: In patients with trauma, rhabdomyolysis (RM) can lead to fatal complications resulting from muscle damage. Thus, RM must be immediately diagnosed and treated to prevent complications. Creatine kinase (CK) is the most sensitive marker for diagnosing RM. However, relying on CK tests may result in delayed treatment, as it takes approximately 1 hour to obtain CK blood test results. Hence, this study investigated whether the neutrophil-to-lymphocyte ratio (NLR) could predict RM at an earlier time point in patients with trauma, since NLR results can be obtained within 10 minutes.

Methods: This retrospective study included 130 patients with severe trauma who were admitted to the emergency room of a tertiary institution between January 2017 and April 2020. RM was defined as a CK level $\geq 1,000 \mathrm{U} / \mathrm{L}$ at the time of arrival. Patients with severe trauma were categorized into non-RM and RM groups, and their characteristics and blood test results were analyzed. Statistical analysis was performed using SPSS version 26.0 for Windows.

Results: Of the 130 patients with severe trauma, 50 presented with RM. In the multivariate analysis, the NLR (odds ratio [OR], 1.252; 95\% confidence interval [CI], 1.1301.386), $\mathrm{pH}$ level (OR, 0.006; 95\% CI, 0.000-0.198), presence of acute kidney injury (OR, 3.009; 95\% CI, 1.140-7.941), and extremity Abbreviated Injury Scale score (OR, 1.819; 95\% CI, 1.111-2.980) significantly differed between the non-RM and RM groups. A receiver operating characteristic analysis revealed that a cut-off NLR value of 3.64 was the best for predicting RM.

Conclusions: In patients with trauma, the NLR at the time of arrival at the hospital is a useful biochemical marker for predicting RM.

Keywords: Rhabdomyolysis; Multiple trauma; Neutrophils; Lymphocytes; Acute kidney injury 


\section{INTRODUCTION}

Rhabdomyolysis (RM) is caused by the breakdown of skeletal muscle and subsequent leakage of its contents into the blood circulation [1]. Common causes of this condition are trauma, overexertion, and drug use, and it can lead to acute kidney injury (AKI), hyperkalemia, cardiac arrhythmia, compartment syndrome, and disseminated intravascular coagulation $[1,2]$. The clinical features of RM include muscle pain, swelling, weakness, tea-colored urine, nausea, and malaise [1]. Creatine kinase (CK) is the most sensitive biomarker of muscle injury, and a baseline $\mathrm{CK}$ value $\geq 1,000 \mathrm{U} / \mathrm{L}$, which is five times the normal upper limit of CK in the blood, is used to diagnose RM [3]. The treatment of RM aims to minimize muscle damage, prevent AKI, and immediately correct life-threatening complications. In particular, proper renal perfusion must be maintained with early and aggressive fluid resuscitation to prevent AKI [3]. A peak CK value $\geq 5,000 \mathrm{U} / \mathrm{L}$ (severe $\mathrm{RM}$ ) indicates severe muscle injury and an elevated risk of developing AKI. However, since CK can reach its peak level within 1-3 days, relying on peak CK levels can result in delays in providing proper treatment [4]. A previous study showed that when the initial CK value was below $900 \mathrm{U} / \mathrm{L}$, the peak CK value did not exceed 5,000 U/L [5]. Therefore, the initial CK value is effective for predicting the peak CK value. It takes approximately 1 hour to obtain CK values from a blood test. Hence, if RM can be predicted before obtaining the $\mathrm{CK}$ test result, appropriate treatment can be provided immediately.

The neutrophil-to-lymphocyte ratio (NLR) can be ob- tained within 10 minutes via a complete blood count examination, and it is used as an indicator of the inflammatory response [6]. The NLR has been used as a predictor of poor prognosis in various conditions, such as infection, infarction, and tumors $[7,8]$. According to Sugama et al. [9], muscle damage leads to neutrophil recruitment and decreased lymphocyte levels during the acute phase. Based on the abovementioned findings, this study aimed to determine whether the NLR could be utilized as an early predictor of RM in patients with acute trauma.

\section{METHODS}

In this retrospective study, the data of patients with trauma who were admitted to the emergency room of a tertiary institution in South Korea between January 2017 and April 2020 were collected. This study was approved by the Institutional Review Board of Chosun University Hospital (IRB No. CHOSUN 2020-03-004). All data were extracted from the electronic medical records of Chosun University Hospital. This study was conducted on injured patients aged $\geq 18$ years who met Chosun University Hospital's criteria for severe trauma team activation, as shown in Table 1, and the severe trauma team was activated when one or more of the criteria were met [10]. The exclusion criteria were patients aged $<18$ years, those for whom excessive time had passed after the accident because of transfer from another hospital, those who died within 24 hours of presenting to the hospital, those with known chronic kidney disease, and those with incomplete

Table 1. Criteria for severe trauma team activation

\begin{tabular}{|lll|}
\hline Physiological parameters & \multicolumn{1}{c|}{ Injury patterns } & Mechanism of injury \\
\hline Systolic blood pressure $<90 \mathrm{mmHg}$ after trauma & Penetrating injuries of the neck and trunk & Fall from height $>3 \mathrm{~m}$ \\
Glasgow Coma Scale score $<9$ after trauma & Gunshot injuries of the neck and trunk & Road traffic accident \\
$\begin{array}{l}\text { Breathing disturbance/need for intubation } \\
\text { after trauma }\end{array}$ & Fractures of at least two proximal bones & Frontal collision with intrusion of more than 50 to \\
& Unstable thorax, unstable pelvic fracture & Changes in velocity of delta $>30 \mathrm{~km} / \mathrm{h}$ \\
& Amputation proximal to heads/feet & Pedestrian/motorcycle collision \\
& Injuries with neurological signs of paraplegia & Death of a passenger \\
& Open cranial injury & Ejection of a passenger \\
& Burns $>20 \%$ & \\
\hline
\end{tabular}


medical records, including blood test results. A total of 228 patients who met the criteria for severe trauma team activation were admitted to Chosun University Hospital emergency room during the study period. Finally, $130 \mathrm{pa}-$ tients were enrolled and 98 were excluded. According to the blood test results performed immediately upon arrival at the hospital, patients with CK levels $\geq 1,000 \mathrm{U} / \mathrm{L}$ were diagnosed with RM. Data were analyzed for a wide range of parameters, including age, sex, Glasgow Coma Scale (GCS) score, blood pressure, heart rate, Acute Physiology and Chronic Health Evaluation II (APACHE II) score, Injury Severity Score (ISS), Abbreviated Injury Scale (AIS) score, trauma mechanism, the presence of acute kidney injury (AKI), continuous renal replacement therapy (CRRT), emergency surgery, mortality, and laboratory values (complete blood count, electrolyte, lactate, blood

Table 2. Patients' characteristics according to RM

\begin{tabular}{|c|c|c|c|c|}
\hline & Total $(n=130)$ & Non RM $(n=80)$ & $\operatorname{RM}(n=50)$ & $p$-value \\
\hline Age (years) & $55.71 \pm 18.09$ & $57.25 \pm 18.36$ & $53.24 \pm 17.58$ & 0.220 \\
\hline Male & $89(68.5)$ & $51(57.3)$ & $38(42.7)$ & 0.144 \\
\hline Female & 41 (31.5) & $29(70.7)$ & $12(29.3)$ & \\
\hline GCS & $11.08 \pm 3.84$ & $11.19 \pm 3.80$ & $10.92 \pm 3.95$ & 0.701 \\
\hline SBP (mmHg) & $91.38 \pm 52.69$ & $97.00 \pm 52.66$ & $82.40 \pm 52.00$ & 0.125 \\
\hline $\mathrm{DBP}(\mathrm{mmHg})$ & $53.54 \pm 30.78$ & $55.37 \pm 29.16$ & $50.60 \pm 33.28$ & 0.392 \\
\hline MAP $(\mathrm{mmHg})$ & $66.04 \pm 37.32$ & $69.10 \pm 36.16$ & $61.14 \pm 38.99$ & 0.238 \\
\hline HR (beat/min) & $88.68 \pm 29.77$ & $88.83 \pm 27.62$ & $88.44 \pm 33.22$ & 0.943 \\
\hline APACHE II & $14.47 \pm 8.56$ & $13.82 \pm 7.62$ & $15.50 \pm 9.88$ & 0.279 \\
\hline ISS & $30.97 \pm 10.55$ & $29.84 \pm 11.36$ & $32.78 \pm 8.92$ & 0.121 \\
\hline Head and neck AIS & $2.18 \pm 1.42$ & $2.29 \pm 1.45$ & $2.00 \pm 1.36$ & 0.262 \\
\hline Face AIS & $1.38 \pm 0.68$ & $1.51 \pm 0.73$ & $1.38 \pm 0.523$ & 0.559 \\
\hline Chest AIS & $3.02 \pm 1.24$ & $3.01 \pm 1.25$ & $3.02 \pm 1.24$ & 0.973 \\
\hline Abdomen AIS & $2.46 \pm 1.31$ & $2.24 \pm 1.29$ & $2.82 \pm 1.27$ & 0.017 \\
\hline Extremity AIS & $2.45 \pm 0.92$ & $2.30 \pm 0.88$ & $2.68 \pm 0.94$ & 0.021 \\
\hline External AIS & $1.18 \pm 0.40$ & $1.22 \pm 0.42$ & $1.10 \pm 0.36$ & 0.076 \\
\hline \multicolumn{5}{|l|}{ Trauma mechanism } \\
\hline Pedestrian TA & $27(20.8)$ & $18(66.7)$ & $9(33.3)$ & \multirow{6}{*}{0.409} \\
\hline Passenger TA & $38(29.2)$ & $24(63.2)$ & $14(36.8)$ & \\
\hline Motorcycle or bicycle TA & $16(12.3)$ & $9(56.3)$ & $7(43.7)$ & \\
\hline Fall & $35(26.9)$ & $20(57.1)$ & $15(42.9)$ & \\
\hline Stab wound & $5(3.8)$ & $5(100)$ & $0(0)$ & \\
\hline Crush injury & $9(6.9)$ & $4(44.4)$ & $5(55.6)$ & \\
\hline AKI & $34(26.2)$ & $15(44.1)$ & $19(55.9)$ & 0.015 \\
\hline CRRT & $16(12.3)$ & $6(37.5)$ & $10(62.5)$ & 0.035 \\
\hline Emergency OP & $96(73.8)$ & $61(63.5)$ & $35(36.5)$ & 0.430 \\
\hline Mortality & $34(26.2)$ & $18(52.9)$ & $16(47.1)$ & 0.230 \\
\hline
\end{tabular}

Values are presented as mean \pm standard deviation or number (\%)

RM: rhabdomyolysis, GCS: Glasgow Coma Scale, SBP: systolic blood pressure, DBP: diastolic blood pressure, MAP: mean arterial pressure, HR: heart rate, ISS: Injury Severity Score, APACHE II: Acute Physiology and Chronic Health Evaluation II, AIS: Abbreviated Injury Scale, TA: traffic accident, AKI: acute kidney injury, CRRT: continuous renal replacement therapy, OP: operation. 
urea nitrogen, and creatinine levels, arterial blood gas analysis findings, and NLR) (Tables 2, 3). Emergency surgery was performed according to the surgeon's discretion. AKI was defined as an increase in serum creatinine level of $>0.3 \mathrm{mg} / \mathrm{dL}$ within 48 hours, a 1.5 -fold elevation from the baseline, or urine output $<0.5 \mathrm{~mL} / \mathrm{kg} / \mathrm{h}$ for 6 hours according to the Kidney Disease Improving Global Outcomes (KDIGO) guidelines. CRRT was provided in cases of kidney injury (a greater than twofold increase in the serum creatinine level, a $>50 \%$ decrease in the glomerular filtration rate, or urine output $<0.5 \mathrm{~mL} / \mathrm{kg} / \mathrm{h}$ for 12 hours) or renal failure (a greater than threefold increase in the serum creatinine level, a $>75 \%$ decrease in the glomerular filtration rate, a serum creatinine level $>4 \mathrm{mg} / \mathrm{dL}$, urine output $<0.3 \mathrm{~mL} / \mathrm{kg} / \mathrm{h}$ for 24 hours, or anuria for $\geq 12$ hours) based on the risk, injury, failure, loss, end-stage kidney disease (RIFLE) criteria or in cases of refractory acidosis or hyperkalemia.

Statistical analysis was performed using SPSS version 26.0 (SPSS, IBM Corp., Armonk, NY, USA). Continuous variables were presented as mean \pm standard deviation, and categorical variables were reported as the frequency and percentage of each item. To evaluate statistical significance, the student's $t$-test was used for continuous variables, and the chi-square test was utilized for categorical variables. Logistic regression analysis was performed using variables that were significant in the univariate analysis. A receiver operating characteristic (ROC) curve was used to determine the appropriate NLR cut-off value for predicting RM. A $p$-value $<0.05$ was considered to indicate statistical significance.

\section{RESULTS}

During the study period (from January 2017 to April 2020), 130 patients with severe trauma who met the criteria were included in the analysis. Based on the initial blood test, 50 patients (38.5\%) were diagnosed with RM (Fig. 1). Significant differences between the patients in the non-RM and RM groups were observed in the abdomen AIS score ( $2.24 \pm 1.29$ vs. $2.82 \pm 1.27 ; p=0.017)$, extremity

Table 3. Univariable analysis of laboratory values

\begin{tabular}{|c|c|c|c|c|}
\hline & Total $(n=130)$ & Non-RM $(n=80)$ & $\mathrm{RM}(n=50)$ & $p$-value \\
\hline WBC $(\times 1,000 / \mu \mathrm{L})$ & $16.62 \pm 11.38$ & $15.91 \pm 13.34$ & $17.77 \pm 7.20$ & 0.367 \\
\hline $\mathrm{Hb}(\mathrm{g} / \mathrm{dL})$ & $12.07 \pm 2.62$ & $12.23 \pm 2.77$ & $11.80 \pm 2.38$ & 0.365 \\
\hline 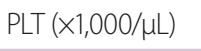 & $205.80 \pm 65.80$ & $213.31 \pm 62.20$ & $193.78 \pm 70.16$ & 0.100 \\
\hline $\mathrm{Na}(\mathrm{mEq} / \mathrm{L})$ & $139.91 \pm 3.87$ & $139.66 \pm 2.58$ & $140.30 \pm 5.33$ & 0.433 \\
\hline $\mathrm{K}(\mathrm{mEq} / \mathrm{L})$ & $3.93 \pm 0.73$ & $3.91 \pm 0.73$ & $3.96 \pm 0.74$ & 0.690 \\
\hline $\mathrm{Cl}(\mathrm{mEq} / \mathrm{L})$ & $104.57 \pm 4.62$ & $104.43 \pm 4.05$ & $104.80 \pm 5.44$ & 0.675 \\
\hline BUN (mg/dL) & $17.40 \pm 8.84$ & $16.69 \pm 9.78$ & $18.53 \pm 7.02$ & 0.250 \\
\hline $\mathrm{Cr}(\mathrm{mg} / \mathrm{dL})$ & $1.25 \pm 1.76$ & $1.33 \pm 2.23$ & $1.12 \pm 0.39$ & 0.523 \\
\hline $\mathrm{pH}$ & $7.34 \pm 0.13$ & $7.36 \pm 0.11$ & $7.30 \pm 0.15$ & 0.016 \\
\hline $\mathrm{PaO}_{2}(\mathrm{mmHg})$ & $74.52 \pm 12.32$ & $76.52 \pm 6.79$ & $73.27 \pm 14.67$ & 0.090 \\
\hline $\mathrm{PaCO}_{2}(\mathrm{mmHg})$ & $36.99 \pm 11.07$ & $37.44 \pm 12.49$ & $36.28 \pm 8.39$ & 0.566 \\
\hline $\mathrm{HCO}_{3}(\mathrm{mmol} / \mathrm{L})$ & $20.43 \pm 4.87$ & $21.08 \pm 4.40$ & $19.37 \pm 5.43$ & 0.047 \\
\hline $\mathrm{BE}(\mathrm{mmol} / \mathrm{L})$ & $-5.86 \pm 5.54$ & $-4.78 \pm 4.81$ & $-7.61 \pm 6.22$ & 0.004 \\
\hline Lactate (mg/dL) & $44.03 \pm 35.45$ & $40.31 \pm 29.98$ & $50.35 \pm 42.79$ & 0.128 \\
\hline NLR & $5.34 \pm 4.71$ & $4.00 \pm 4.01$ & $7.47 \pm 4.91$ & $<0.001$ \\
\hline
\end{tabular}

Values are presented as mean \pm standard deviation.

RM: rhabdomyolysis, WBC: white blood cell, Hb: hemoglobin, PLT: platelet, BUN: blood urea nitrogen, Cr: creatinine, BE: base excess, NLR: neutrophil-to-lymphocyte ratio. 
AIS score ( $2.30 \pm 0.88$ vs. $2.68 \pm 0.94 ; p=0.021)$, the presence of AKI (15 [44.1\%] vs. 19 [55.9\%]; $p=0.015)$, and CRRT (6 [37.5\%] vs. 10 [62.5\%]; $p=0.035$ ) (Table 2). However, the two groups did not significantly differ according to age, sex, GCS score, blood pressure, heart rate, APACHE II score, ISS score, AIS score (head and neck, face, chest, and external), trauma mechanism, emergency surgery status, and mortality (Table 2). Based on the initial blood test results, the two groups significantly differed in terms of $\mathrm{pH}$ level $(p=0.016)$, bicarbonate level $(p=0.047)$, base excess $(p=0.004)$, and NLR $(p<0.001)$ (Table 3$)$. A multivariate logistic regression analysis was performed using variables with significant differences in the univariate analysis

Table 4. Association of rhabdomyolysis with clinical variables by multivariable logistic regression analysis

\begin{tabular}{|lccc|}
\hline Variables & OR & $95 \% \mathrm{Cl}$ & $\boldsymbol{p}$-value \\
\hline NLR & 1.252 & $1.130-1.386$ & $<0.001$ \\
$\mathrm{pH}$ & 0.006 & $0.000-0.198$ & 0.004 \\
AKI & 3.009 & $1.140-7.941$ & 0.026 \\
Extremity AIS & 1.819 & $1.111-2.980$ & 0.017 \\
\hline
\end{tabular}

OR: odds ratio, Cl: confidence interval, NLR: neutrophil-to-lymphocyte ratio, AKI: acute kidney injury, AIS: Abbreviated Injury Scale.

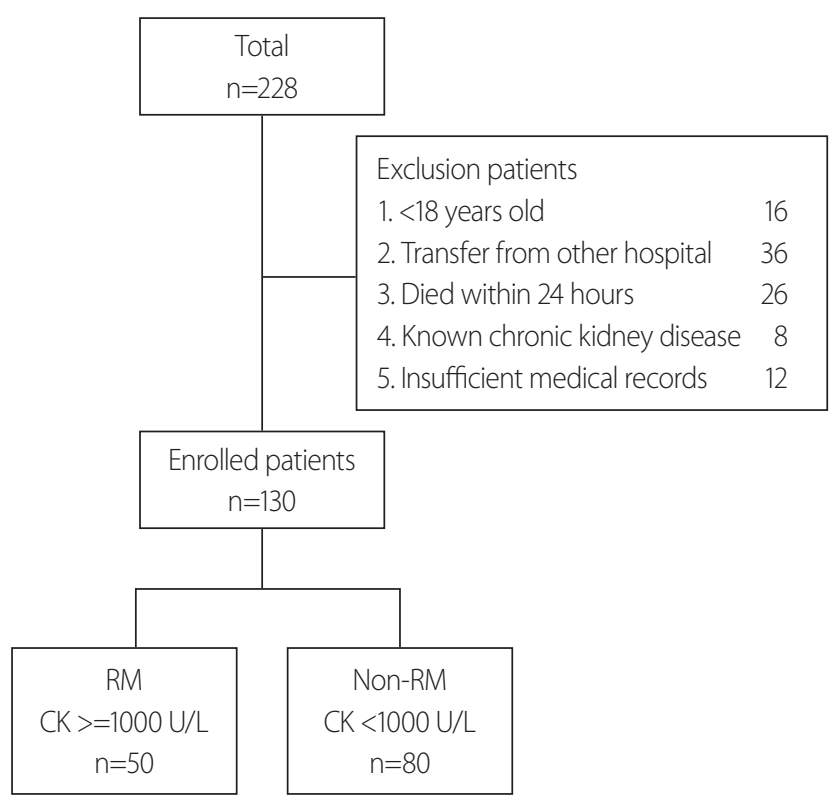

Fig. 1. Flow diagram of patient enrollment. RM; rhabdomyolysis, CK; creatine kinase.
(Tables 2, 3). The results showed significant between-group differences in NLR (odds ratio [OR], 1.252; 95\% confidence interval [CI], 1.130-1.386; $p<0.001), \mathrm{pH}$ level (OR, 0.006; 95\% CI, 0.000-0.198; $p=0.004)$, presence of AKI (OR, 3.009; 95\% CI, 1.140-7.941; $p=0.026)$, and extremity AIS score (OR, 1.819; 95\% CI, 1.111-2.980; $p=0.017$ ) (Table 4). A ROC analysis was performed to determine the most appropriate cutoff value of the NLR for diagnosing RM. The best cutoff value was found to be 3.64 (sensitivity, 0.780; specificity, 0.662), and the area under the curve was 0.741 (Fig. 2). Using the chi-square test, an analysis of the non-RM and RM groups based on an NLR value of $3.64(p<0.001)$ revealed that the NLR was a sig-

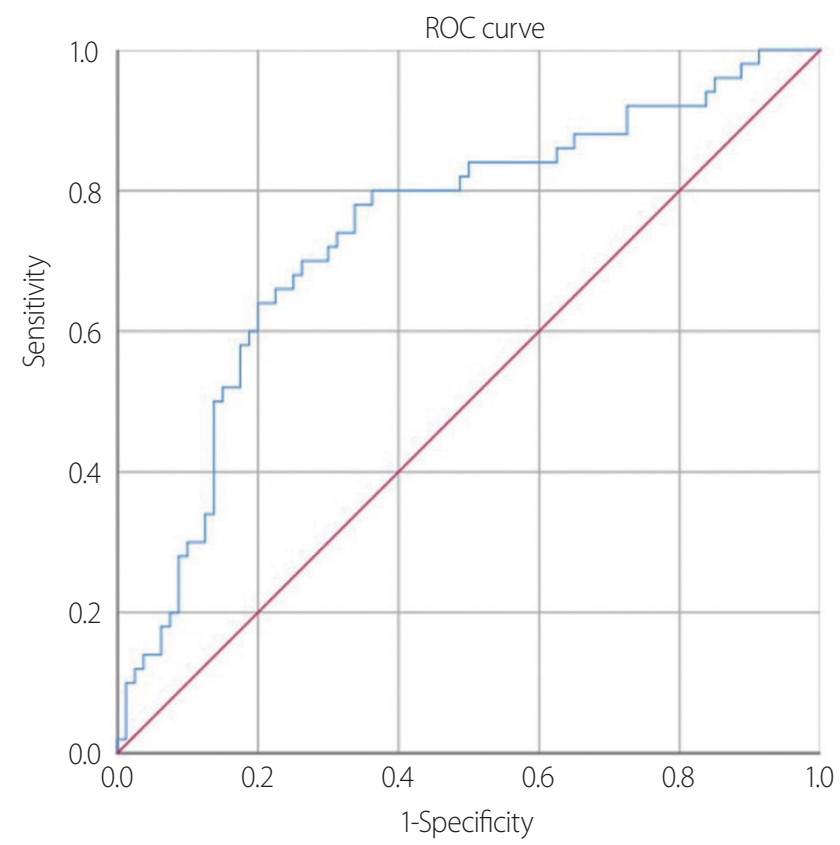

Fig. 2. Receiver operating characteristic curve for the neutrophil-to-lymphocyte ratio in predicting rhabdomyolysis in patients with severe trauma (area under the curve $=0.741$ ).

Table 5. Correlations between NLR and rhabdomyolysis

\begin{tabular}{|lcccc|}
\hline & Non-RM & RM & Total & p-value \\
\hline Low NLR (<3.63) & $53(40.7)$ & $11(8.5)$ & $64(49.2)$ & \\
High NLR (>3.64) & $27(20.8)$ & $39(30.0)$ & $66(50.8)$ & $<0.001$ \\
& $80(61.5)$ & $50(38.5)$ & $130(100)$ & \\
\hline
\end{tabular}

Variables are presented as number (\%).

RM: rhabdomyolysis, NLR: neutrophil-to-lymphocyte ratio. 
nificant biomarker for predicting RM (Table 5).

\section{DISCUSSION}

In this study, patients with trauma who visited the emergency room of Chosun University Hospital were diagnosed with RM based on their initial CK values. We assessed whether other initial blood test values could be used to predict RM. Result showed that the NLR value and $\mathrm{pH}$ level significantly differed between the $\mathrm{RM}$ and non-RM groups. When muscle damage occurs, leukocytes (particularly neutrophils) are activated, and they move to the site of damage and act as early responders $[11,12]$. In a previous study, the blood neutrophil count significantly increased within 6 hours after muscle damage in patients with a $C K$ value $\geq 1,000 \mathrm{U} / \mathrm{L}$ compared with patients with a CK value $<1,000 \mathrm{U} / \mathrm{L}$ [11]. In another study, the neutrophil count increased within 3 hours of muscle damage, while the lymphocyte count decreased [9]. The NLR only increases when the neutrophil count increases and/or the lymphocyte count decreases. An elevated NLR is caused by an increase in serum cortisol levels, which is a stress response that can be used as a biochemical index to determine the balance between the body's immune system and immune suppression during inflammation [6]. An elevated NLR is an indirect and simple indicator of the imbalance between these two responses, and it is caused by a combination of increased neutrophil production and lymphocyte cell death due to stresses (e.g., infection and trauma) [13]. Taken together, if RM occurs due to muscle damage, the NLR will increase with an initial elevation in the neutrophil count and reduction in the lymphocyte count due to an inflammatory reaction. In accordance with the aforementioned studies, the results of this study confirm that there is an increase in the initial NLR in patients with RM who present with significant muscle damage.

Metabolic acidosis, which is caused by oxygen depletion in the involved tissue or organ, is an important complication of RM [14]. In patients with RM, when muscles are destroyed and toxic substances are released from the cells, local edema and ischemia occur, and ischemia results in energy depletion and metabolic acidosis [15]. In this study, the pH level was significantly lower (reflecting acidosis) in the RM group with more severe muscle damage than in the non-RM group.

This study showed that abdominal AIS and extremity AIS scores significantly differed according to initial RM status in the univariate analysis; however, the abdominal AIS score did show a significant relationship with RM in the multivariate analysis. According to the results of a recent study, the ISS, chest AIS, abdominal AIS, and extremity AIS scores significantly differed in a univariate analysis performed according to severe RM (peak CK value $\geq 5,000$ $\mathrm{U} / \mathrm{L})$. However, the only significant relationship identified in the multivariate analysis was the extremity AIS score [16]. Moreover, that previous study targeted only patients with blunt trauma, whereas this study included all severe trauma patients, and the previous study used a different cutoff value for CK (1,000 vs. 5,000 U/L). These factors may have caused the discrepancies in the findings of the univariate analysis between the two studies. However, the multivariate analysis revealed similar results, and the consistent relationship of the extremity AIS score with RM may be less affected by other variables because there are numerous skeletal muscles in the extremities. To predict the long-term complications and risk factors of RM, a previous study used the peak CK level as a cutoff value to diagnose RM, and found this value to be more accurate than other predictors. However, CK levels take 1-3 days to reach their peak, which can result in a delay in providing proper treatment. Therefore, this study is meaningful in that it identified a factor that can predict RM at its early stages and can facilitate the immediate provision of appropriate treatment.

AKI is a potentially fatal complication that can occur in patients with RM due to trauma. AKI is a complex syndrome characterized by a decrease in renal function that is defined based on the RIFLE and Acute Kidney Injury Network classifications. Moreover, the KDIGO classification is often used [17]. In this study, AKI was diagnosed based on the KDIGO classification. The incidence of AKI due to RM varies between $13 \%$ and $50 \%$ according to previous studies, and 19 of 50 patients (38\%) had AKI due to RM in our study. In this condition, when muscle injury occurs, myoglobin accumulates in the kidney, leading to intrarenal vasoconstriction, direct and ischemic tubule 
injury, and tubular obstruction, which result in AKI [18]. When AKI occurs, volume overload, electrolyte disorders, uremic complications, and drug toxicity may develop [19], and the incidence of AKI increases with elevated blood CK values [20]. According to Arulselvi et al. [21], there was a significant difference in the relationship between the CK level and the incidence of RM on the first, third, and fifth days after trauma. This result was consistent with the finding of our study that the CK value in the early stage of trauma was significantly correlated with the occurrence of AKI. Based on these results, if RM caused by trauma is immediately diagnosed after a patient presents to the hospital, treatment can be immediately started and the occurrence of AKI will then be prevented. The treatment of RM patients in the acute phase should aim to preserve renal function and restore metabolic derangements. The early administration of large volumes of normal saline is the most important intervention to prevent AKI. Volume expansion increases renal blood flow and glomerular filtration [22]. However, in older patients with cardiopulmonary risk factors, cautious observation is necessary because high-volume fluid therapy can exacerbate congestive heart failure and cause pulmonary edema [22]. In relation to this, the risk of RM should be predicted upon arrival at the hospital should be predicted to provide adequate fluid therapy to patients with trauma.

This study had several limitations that should be acknowledged. Specifically, it was conducted at a single center, the number of participants was small, and peak CK values could not be analyzed, as several patients did not undergo serial testing for CK values after the initial assessment. Therefore, factors associated with the peak CK value and whether it predicted complications could not be evaluated. In addition, other factors that could cause acidosis (hypovolemia and drug use) were not considered. Hence, a prospective study considering these factors should be conducted in the future.

\section{CONCLUSION}

In patients with trauma, RM can cause fatal complications. Hence, immediate diagnosis and treatment are important. However, the most sensitive CK value for
RM diagnosis can be only obtained after approximately 1 hour. In contrast, since NLR results can be obtained within 10 minutes via a complete blood count, the NLR can help predict RM at an earlier time point in patients with trauma. Then, appropriate treatment can be provided. Therefore, the NLR is a predictive factor of RM in patients with trauma, and immediate treatment of RM can reduce complications, including AKI.

\section{REFERENCES}

1. Sauret JM, Marinides G, Wang GK. Rhabdomyolysis. Am Fam Physician 2002;65:907-12.

2. Rodríguez E, Soler MJ, Rap O, Barrios C, Orfila MA, Pascual J. Risk factors for acute kidney injury in severe rhabdomyolysis. PLoS One 2013;8:e82992.

3. Zimmerman JL, Shen MC. Rhabdomyolysis. Chest 2013;144:1058-65.

4. Huerta-Alardín AL, Varon J, Marik PE. Bench-to-bedside review: rhabdomyolysis -- an overview for clinicians. Crit Care 2005;9:158-69.

5. Assanangkornchai N, Akaraborworn O, Kongkamol C, Kaewsaengrueang K. Characteristics of trauma patients with creatine kinase elevation. Crit Care 2015;19(Suppl 1):P282.

6. Zahorec R. Ratio of neutrophil to lymphocyte counts--rapid and simple parameter of systemic inflammation and stress in critically ill. Bratisl Lek Listy 2001;102:5-14.

7. Hwang SY, Shin TG, Jo IJ, Jeon K, Suh GY, Lee TR, et al. Neutrophil-to-lymphocyte ratio as a prognostic marker in critically-ill septic patients. Am J Emerg Med 2017;35:234-9.

8. Lee JB, Lee SH, Yung SJ, Ryu SY, Choi SW. Kim HJ, et al. Neutrophil-to-lymphocyte ratio as a predictor of aspiration pneumonia in drug intoxication patients. J Korean Soc Clin Toxicol 2018;16:61-7.

9. Sugama K, Suzuki K, Yoshitani K, Shiraishi K, Kometani T. IL17 , neutrophil activation and muscle damage following endurance exercise. Exerc Immunol Rev 2012;18:116-27.

10. Frink M, Lechler P, Debus F, Ruchholtz S. Multiple trauma and emergency room management. Dtsch Arztebl Int 2017;114:497503.

11. Isaacs AW, Macaluso F, Smith C, Myburgh KH. C-reactive protein is elevated only in high creatine kinase responders to muscle damaging exercise. Front Physiol 2019;10:86. 
12. Paulsen G, Crameri R, Benestad HB, Fjeld JG, Mørkrid L, Hallén J, et al. Time course of leukocyte accumulation in human muscle after eccentric exercise. Med Sci Sports Exerc 2010;42:75-85.

13. Taneja R, Parodo J, Jia SH, Kapus A, Rotstein OD, Marshall JC. Delayed neutrophil apoptosis in sepsis is associated with maintenance of mitochondrial transmembrane potential and reduced caspase-9 activity. Crit Care Med 2004;32:1460-9.

14. Zhang MH. Rhabdomyolosis and its pathogenesis. World J Emerg Med 2012;3:11-5.

15. Keltz E, Khan FY, Mann G. Rhabdomyolysis. The role of diagnostic and prognostic factors. Muscles Ligaments Tendons J 2014;3:303-12.

16. Park JY, Kim MJ, Lee JG. Early predictive values for severe rhabdomyolysis in blunt trauma. J Trauma Inj 2019;32:26-31.

17. Gameiro J, Agapito Fonseca J, Jorge S, Lopes JA. Acute kidney injury definition and diagnosis: a narrative review. J Clin Med
2018;7:307.

18. Bosch X, Poch E, Grau JM. Rhabdomyolysis and acute kidney injury. N Eng J Med 2009;361:62-72.

19. Levey AS, James MT. Acute kidney injury. Ann Intern Med 2017;167:ITC66-80.

20. Nawaporn A, Osaree A, Chanon K, Khanitta K. Characteristics of creatine kinase elevation in trauma patients and predictors of acute kidney injury. JACME 2017;7:54-60.

21. Arulselvi S, Deepti S, Vivek T, Arbind KP, Venencia A, Ravindra MP. Evaluation of serum creatine kinase and urinary myoglobin as markers in detecting development of acute renal failure in severely injured trauma patients. ISRN Emergency Medicine 2013;2013:1-8.

22. Zutt R, van der Kooi AJ, Linthorst GE, Wanders RJ, de Visser M. Rhabdomyolysis: review of the literature. Neuromuscul Disord 2014;24:651-9. 